\title{
Micromechanical Models for Bending Behavior of Woven Composites
}

\author{
Ö. Soykasap* \\ Department of Engineering, \\ University of Cambridge \\ Trumpington Street, Cambridge, CB2 1PZ, UK
}

\begin{abstract}
Thin woven composites have been popular for space structures due to the symmetrical and balanced properties. Although in-plane properties of these materials can be calculated accurately using the classical lamination theory (CLT), the corresponding bending properties lack any accuracy for one or two-ply woven laminates. Experiments on thin laminates made from woven composites disagree with the estimates of bending stiffness and strains using CLT. Such estimates can result in errors of up to $200 \%$ in the maximum bending strains or stresses, and up to $400 \%$ in the bending stiffnesses. This is because CLT assumes that the fibers and the matrix are uniformly distributed in each lamina, and relies on this uniformity in the integration of the transformed laminate stiffnesses over the thickness of the laminate. However, a thin laminate made from fabrics in fact consists of bundles of fibers that are typically much thinner than the overall thickness of the laminate; these bundles are not homogenous through the thickness. This paper presents micromechanical models for bending behavior of woven composites considering the fiber bundles and the matrix and their interactions. Finite element models are developed to estimate the bending properties of plain weave composites. The results are compared to experimental data, showing very good agreement particularly for a lamina.
\end{abstract}

\section{Introduction}

Thin woven composites have been popular for space structures due the symmetrical and balanced properties coupled with very good handling and damage characteristics. Their use in ultra-thin deployable structures is of current interest, where it is envisaged that a laminate will consist of only a small number of plies.

Woven composites have different style such as plain weave, twill, satin etc. Material properties depends on the number of fibers in warp and weft directions as well as the type of weave. The type of the weave is also important because it determines processability of the composite, such as drapeability over a curved surface. In plain weave style, each warp fiber passes alternately over and under each fill (weft) fiber (see Figure 1). This papers studies bending behavior of plain weave composites.

A schematic cross-section of a plain weave lamina is shown in Figure 2, where $h$ and $L$ are the amplitude of the wave formed by the centerline of the fibers and their wave length. An image of the cross-section of plain weave laminate, obtained with an optical microscope, is shown in Figure 3.

The estimation of the mechanical properties of these thin laminates is crucial for the design of future deployable structures. Although the in-plane properties of a thin laminate can be calculated with good accuracy

\footnotetext{
*Senior Research Associate, E-mail: os239@eng.cam.ac.uk, Member AIAA.
}

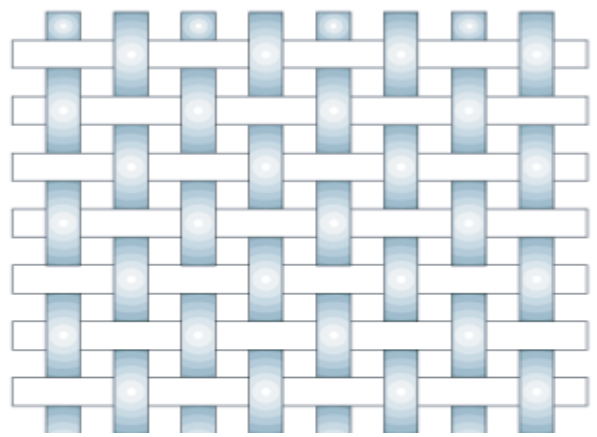

Fig. 1 Plain-weave style.

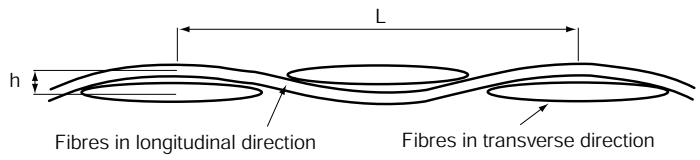

Fig. 2 Basic definitions of woven fiber microstructure.

using classical lamination theory (CLT), the bending properties of these materials lack any accuracy for one or two-ply woven laminates. Experiments on thin laminates made from woven composites disagree with the predictions of bending stiffness and strains using classical lamination theory [1]. The bending properties obtained from CLT can result in errors of up to $200 \%$ in the maximum bending strains or stresses, and up to $400 \%$ in the bending stiffnesses. The problem is that CLT assumes that the fibers and the matrix are 


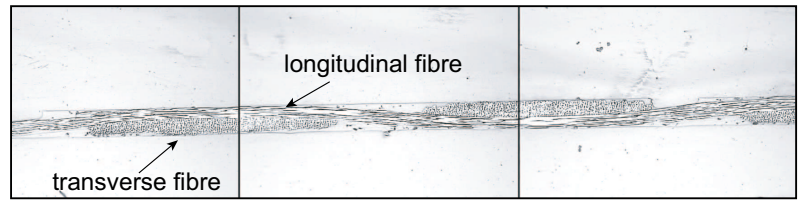

(a)

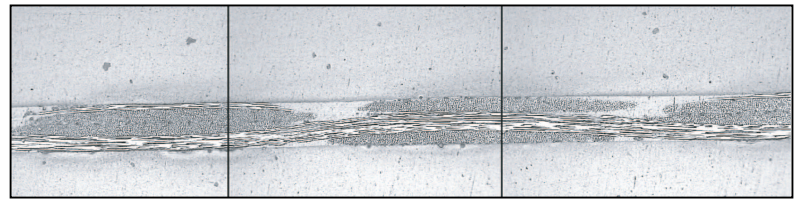

(b)

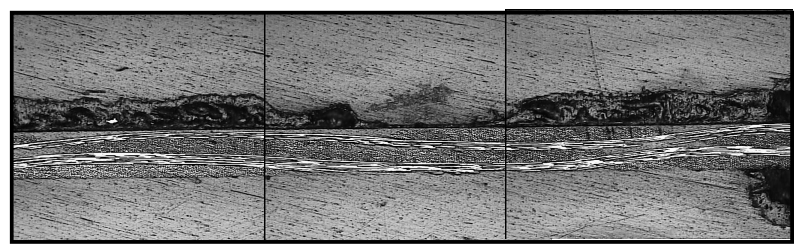

(c)

$1 \mathrm{~mm}$

Fig. 3 Optical microscope image of microstructure of (a) 1-ply T300/LTM45, (b) 2-ply T300/LTM45 (fibers out of phase), (c) 2-ply T300/LTM45 (fibers in phase).

uniformly distributed in each lamina, uses in-plane material properties to analyze the macromechanical behavior of a laminate, and relies on this uniformity in the integration of the transformed laminate stiffnesses over the thickness of the laminate [2]. However, it is clear from Figure 3 that a thin laminate made from fabrics in fact consists of bundles of fibers (called yarn) that are typically much thinner than the overall thickness of the laminate; these bundles are not homogenous through the thickness and hence CLT needs to be used with great care in the present case (if is not altogether abandoned, in favour of a detailed micromechanical model).

This paper presents micromechanical models for bending behavior of woven composites considering the fiber bundles, the matrix, and their interactions. Finite element models are developed to predict the bending properties of plain weave T300/LTM45 composite. Initially curved beam elements are used to model each fiber bundle. Interlaced bundles are connected at the crossover points using rigid beam connectors, representing the resin interface. The cross-section of each beam is approximated by a rectangle of area equal to that of the bundles observed in micrographs of the laminate cross-section. The material properties of the beams are estimated using the fiber volume fraction. A geometrically non-linear analysis of the resulting "space frame" is carried out, in order to investigate the behaviour of one, two, and three-ply woven composites. The results are compared to experimental data, showing very good agreements particularly for a single ply.

\section{Material Properties}

In the present study, T300/LTM45 plain weave composite manufactured by Brookhouse Paxford, UK, is studied. Plain weave is made of $1 \mathrm{~K}$ T300 fibers, has an areal weight of $94 \mathrm{gsm}$. LTM45 cures at a temperature of $60^{\circ} \mathrm{C}$ for 9 hours. Material properties of fiber and matrix are given in Table 1.

Table 1 Material properties of T300 and LTM45.

\begin{tabular}{|c|c|c|}
\hline Property & T-300 & LTM45 \\
\hline $\mathrm{E}_{1}(\mathrm{GPa})$ & 230 & 3.1 \\
\hline $\mathrm{E}_{2}(\mathrm{GPa})$ & 14 & 3.1 \\
\hline $\mathrm{G}_{12}(\mathrm{GPa})$ & 9 & 1.1 \\
\hline$v_{12}$ & 0.2 & 0.41 \\
\hline Density $\left(\mathrm{kg} / \mathrm{m}^{3}\right)$ & 1760 & 1230 \\
\hline Tensile Strength $(\mathrm{MPa})$ & 3530 & - \\
\hline Maximum strain $(\%)$ & 1.5 & - \\
\hline
\end{tabular}

Based on measurements from micrographs, T300/LTM45 plain weave lamina has the amplitude of the wave formed by the centreline of the fibers, $h=0.055 \mathrm{~mm}$, and wave length $L=2.75 \mathrm{~mm}$.

The thickness of each yarn is taken to be half of the thickness of the composite. The cross-section of the beam is approximated as a rectangle $1.375 \mathrm{~mm} \times$ $0.055 \mathrm{~mm}$, taking the equivalent area of a lenticularshaped yarn.

Material properties of each yarn are based on the fiber volume fraction in the yarn, and estimated using rule of mixtures. T300 filament has a diameter of $6.9 \mu \mathrm{m}$, and the total cross-sectional area of $1 \mathrm{~K}$ fibers is $A_{f}=3.75 \times 10^{-2} \mathrm{~mm}^{2}$. Fiber volume fraction $V_{f}$ in the yarn is then calculated as follows

$$
V_{f}=\frac{A_{f}}{A_{y}}=0.5
$$

where $A_{y}=1.375 \times 0.055 \mathrm{~mm}^{2}$ is the cross-sectional area of the yarn. Using the material properties given in Table 1 longitudinal modulus, transverse modulus, shear modulus and Poisson's ratio of the yarn are calculated respectively as follows

$$
\begin{gathered}
E_{1}^{y}=V_{f} E_{1 f}+V_{m} E_{m}=115.6 \mathrm{GPA} \\
E_{2}^{y}=\frac{E_{1 f} E_{m}}{V_{f} E_{m}+V_{m} E_{1 f}}=5.1 \mathrm{GPA} \\
G_{12}^{y}=\frac{G_{12 f} G_{m}}{V_{f} G_{m}+V_{m} G_{11 f}}=1.96 \mathrm{GPA} \\
\nu_{12}^{y}=V_{f} \nu_{12 f}+V_{m} \nu_{m}=0.305
\end{gathered}
$$

\section{Micromechanical Models}

A number of finite element models have been developed to estimate material properties of woven composites. For example Fujita et al. [3] and Dano et 
al. [4]) used beam elements to study in-plane properties of woven composites; recently Bednarcyk and Arnold [5] developed three-dimensional repeating unit cell model to study woven composites; more recently Page et al. [6] developed two-dimensional FE model to study damage properties. In the present study, one, two and three-ply woven laminates are modelled using finite element (FE) program Abaqus [7]. Initially curved beams are used to model each yarn. The curved shape of the beam is taken to be a sine curve. Interlaced yarns are connected at the crossover points using beam connectors. Geometrically nonlinear analysis is carried out. Material properties of the beams are approximated using fiber volume fraction.

Figure 4 shows a plain weave lamina with 6 by 15 units (i.e. 7 yarns in warp and 16 yarns in weft direction). The ends of the model are constrained to be rigid using multi point constraints for the cross-over nodes at the ends, that are connected to a reference point placed in the mid-plane of the structure at the either ends. The model is meshed using 2-node cubic beam elements B33.

Interlaced yarns are connected at the crossover points using rigid beam connectors, representing the bonding between two interlacing yarns, and transferring loads from one yarn to another. The beam connector imposes kinematic constraints between two nodes $a$ and $b$; (i) the distance between the two nodes is kept constant, (ii) the position of the second node remain on a line defined by the orientation of the first node and the initial position of the second node, and (iii) their local directions are aligned as seen in Figure 5 .

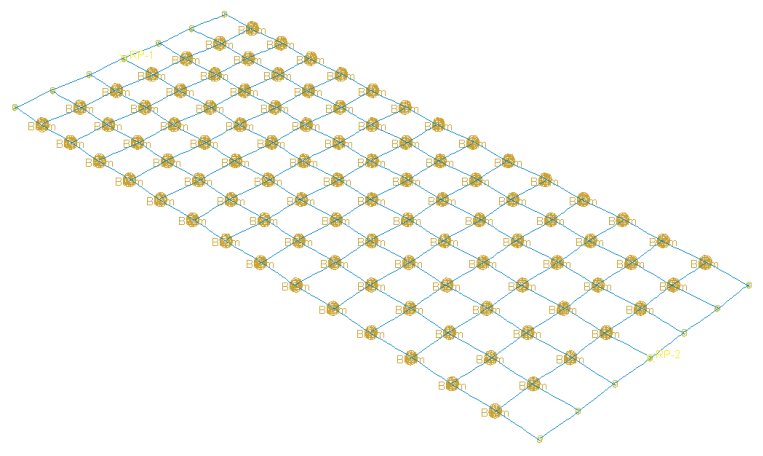

Fig. 4 Plain weave lamina model (single ply: 6 by 15 units).

\section{Stretching Simulation}

In order to verify FE model, stretching simulation is carried out since in-plane properties are expected to be accurate. For stretching simulation, one end of the model is assumed to be fixed while the other end of the model is subjected to prescribed displacements of the reference point at the other end. In order to

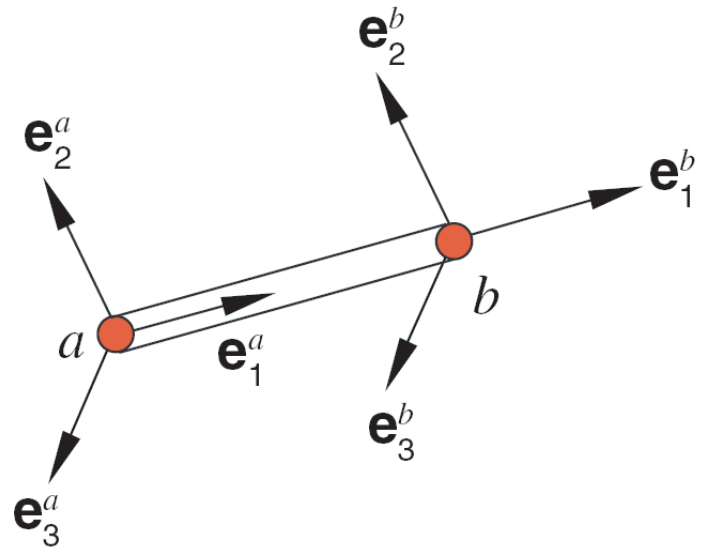

Fig. 5 Beam connector definition for nodes $a$ and b.

see the effect of model size on the material properties, two different models, 6 by 7 unit cell $(9.625 \mathrm{~mm}$ wide and $9.625 \mathrm{~mm}$ long) and 6 by 15 unit cell $(9.625$ $\mathrm{mm}$ wide and $20.625 \mathrm{~mm}$ long) are considered. Nonlinear analysis results are compared in Figure 6, in which stress and strain values are obtained considering the reference points at the ends of the model and global dimensions of the model. Figure 7 shows midsection strains of the yarns when the model is subject to $1 \%$ extension. The longitudinal yarns are subject to uniform high strains whereas the strains of transverse yarns are insignificant. The longitudinal yarns are under combined effect of bending and stretching due to the crimp.

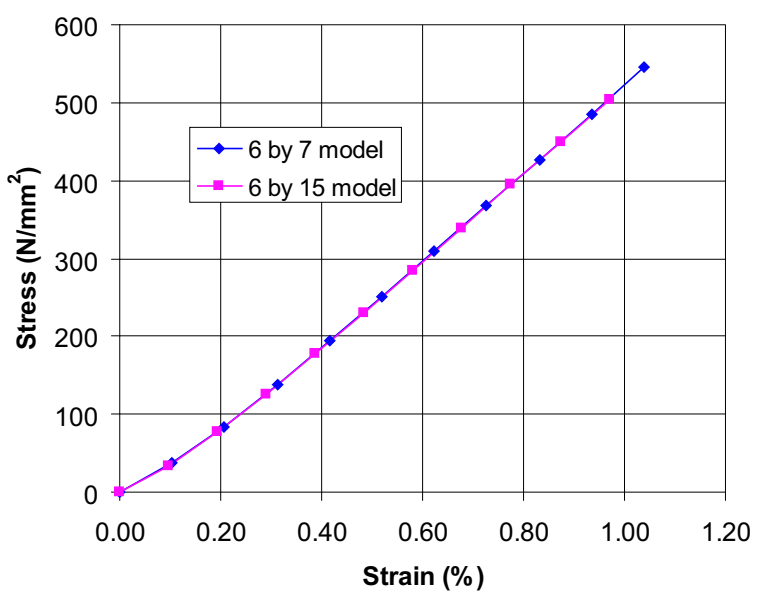

Fig. 6 Stretching simulation of one-ply models.

According to simulations there is an initial nonlinearity due to the crimp of the fibers, and increased model size does not play significant role. Ignoring initial nonlinearity elastic modules of the one-ply com- 


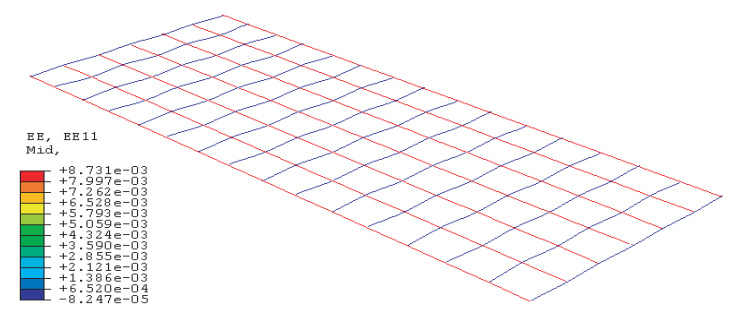

Fig. 7 Strains of 6 by 15 model subject to $1 \%$ extension.

posite are compared in Table 2. As expected longitudinal stiffness is captured very well with only $1 \%$ difference from the manufacturer's data. A simple estimate of the elastic modulus can be calculated using the rule of mixtures as follows

$$
E_{1}=0.5 V_{f} E_{1 f}+V_{m} E_{m}
$$

where the coefficient of 0.5 in the first term on the right hand side is due to the fact that half of the fibers are in transverse direction.

Table 2 Comparison of elastic modulus of one ply model.

\begin{tabular}{|l|c|}
\hline & $\mathbf{E}_{1}(\mathbf{G P a})$ \\
\hline 6 by 7 model & 55.7 \\
\hline 6 by 15 model & 55.4 \\
\hline Manufacturer's data & 56.0 \\
\hline Rule of mixtures & 57.8 \\
\hline
\end{tabular}

\section{Bending Simulation}

For bending simulation prescribed rotations (which are equal and opposite) are applied at both ends via the reference points. Then geometrically nonlinear analysis is carried out. The simulations of one, two and three-ply models are studied, and discussed in what follows.

In order to compare the simulation results to those of CLT, well-known equations of CLT are also given below.

According to CLT the constitutive equations for a thin plate (see Figure 8), in terms of stress and strain resultants are

$$
\left\{\begin{array}{l}
N \\
\cdots \\
M
\end{array}\right\}=\left[\begin{array}{ccc}
A & \vdots & B \\
\cdots & & \cdots \\
B & \vdots & D
\end{array}\right]\left\{\begin{array}{c}
\epsilon^{0} \\
\cdots \\
\kappa
\end{array}\right\}
$$

where $N$ force resultants, $M$, moment resultants, $A$ extensional stiffness, $B$ in-plane/flexture coupling stiffness, $D$ bending stiffness, $\epsilon^{0}$ mid-plane strains, and $\kappa$ mid-plane curvatures.

The strains at any point in the laminate are calculated using mid-plane strains and the curvatures as follows:

$$
\left[\begin{array}{c}
\epsilon_{x} \\
\epsilon_{y} \\
\epsilon_{x y}
\end{array}\right]=\left[\begin{array}{c}
\epsilon_{x}^{o} \\
\epsilon_{y}^{o} \\
\epsilon_{x y}^{o}
\end{array}\right]+z\left[\begin{array}{c}
\kappa_{x} \\
\kappa_{y} \\
\kappa_{x y}
\end{array}\right]
$$

It is assumed that the laminate is symmetric $B=0$, and is subject to pure bending and large curvature $\kappa_{x}$, i.e. $N_{x}=N y=N_{x y}=M_{y}=M_{x y}=0$, and $M_{x} \neq 0$. Ignoring $\kappa_{y}$ and $\kappa_{x y}$ Eqs. 7 and 8 reduce to

$$
\begin{gathered}
M_{x}=D_{11} \kappa_{x} \\
\epsilon_{x}=z \kappa_{x}
\end{gathered}
$$

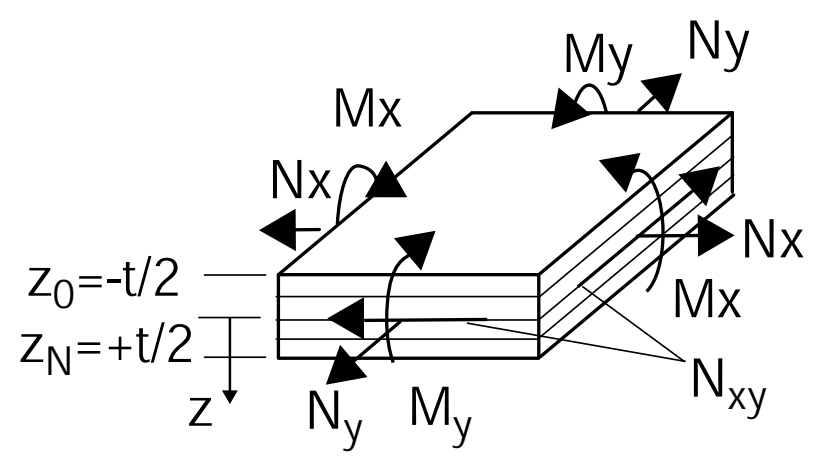

Fig. 8 Lay-up definition of a laminate.

\section{Single Ply}

Single-ply models consist of 6 by 7 units and 6 by 15 units. Figures 9 and 10 show maximum strain and stress values of one-ply model ( 6 by 15 units) which is $180^{\circ}$ bent, respectively. Since the extreme strain and stress values occur at the outer fiber of the beam, top-right corner of the rectangular cross-section is considered. According to the simulation warp fibers are subject to high strains and stresses whereas the strains and stresses of weft fibers are not significant. Variation of longitudinal strains and stresses along warp yarns is almost uniform.

Figures 11 and 12 show moment per unit width versus curvature and maximum strain versus curvature obtained from both FE model ( 6 by 15) and CLT, respectively. Linear behavior of the model is observed. Bending stiffness and minimum bending radius of the models are compared to experimental data [8] as well as to those of CLT in Table 3. Minimum bending radius of the models corresponds to the maximum strains of $1.5 \%$, which is the ultimate strain of the fiber.

The experimental data give a range for different specimen size. Both models ( 6 by 7 and 6 by 15) yield similar results which are in very agreement with the experimental values. When compared to average experimental data, there is only $3 \%$ difference in bending stiffness, and $4 \%$ difference in minimum bend radius. Clearly CLT overestimates both bending stiffness and minimum bend radius by a factor of 3.9 and 2 , respectively. 

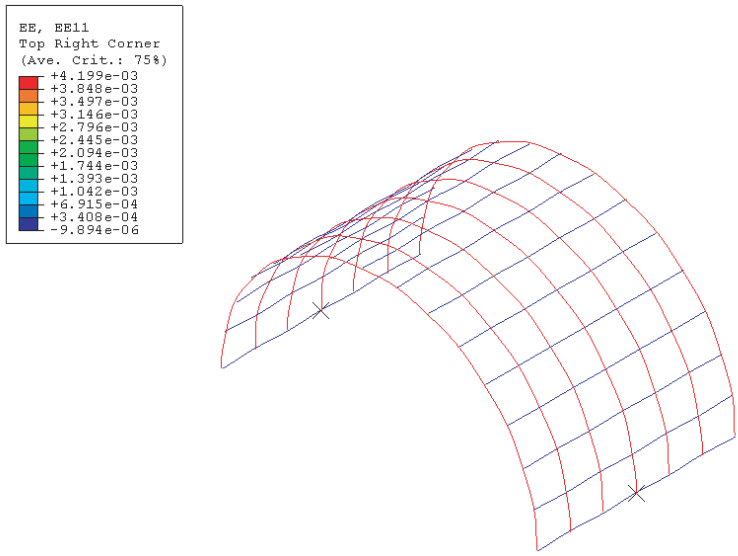

Fig. 9 Strains of $180^{\circ}$ bent model (single ply: 6 by 15 units).
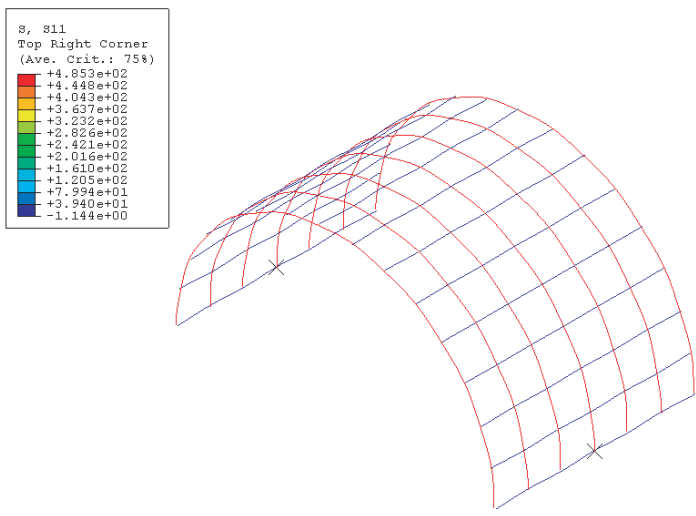

Fig. 10 Stresses of $180^{\circ}$ bent model (single ply: 6 by 15 units).

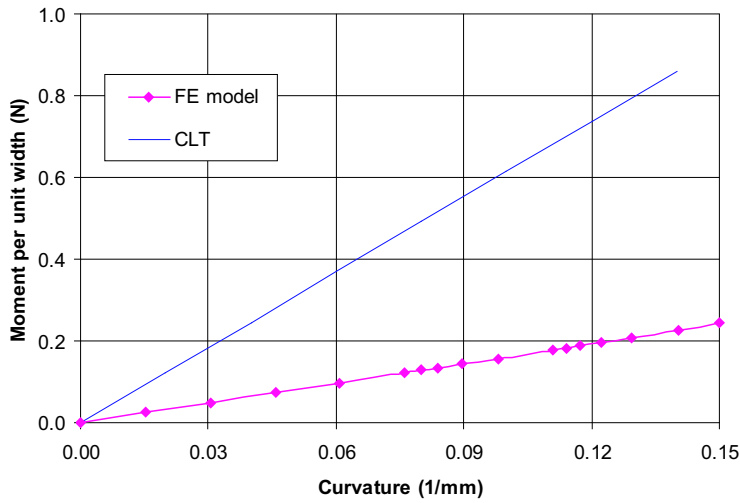

Fig. 11 Moment per unit width versus curvature (single ply: 6 by 15 units).

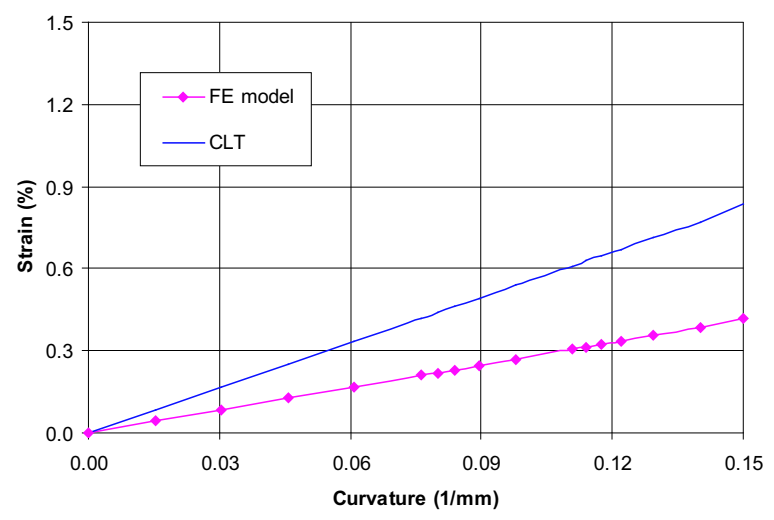

Fig. 12 Strain versus curvature (single ply: 6 by 15 units).

Table 3 Bending stiffness and minimum radius of curvature of one ply.

\begin{tabular}{|c|c|c|}
\hline Single ply & $\begin{array}{c}\mathbf{D}_{11} \\
(\mathbf{N m m})\end{array}$ & $\begin{array}{c}\mathbf{R}_{\min } \\
(\mathbf{m m})\end{array}$ \\
\hline 6 by 7 model & 1.6 & 1.85 \\
\hline 6 by 15 model & 1.6 & 1.84 \\
\hline Experiment (Ref. 8) & $1.54 \sim 1.57$ & $1.69 \sim 1.85$ \\
\hline CLT & $6.23 *$ & $3.67^{\dagger}$ \\
\hline
\end{tabular}

\section{Two Plies}

For a two-ply laminate, the alignment of the warp or weft fibers from each ply has a significant effect on bending properties of the material. Two extreme cases are studied: first the pair of either warp or weft fibers are in phase (i.e. parallel to each other), next the pair of either warp or weft fibers are out of phase (i.e. opposite direction of each other) as in Figure 13.

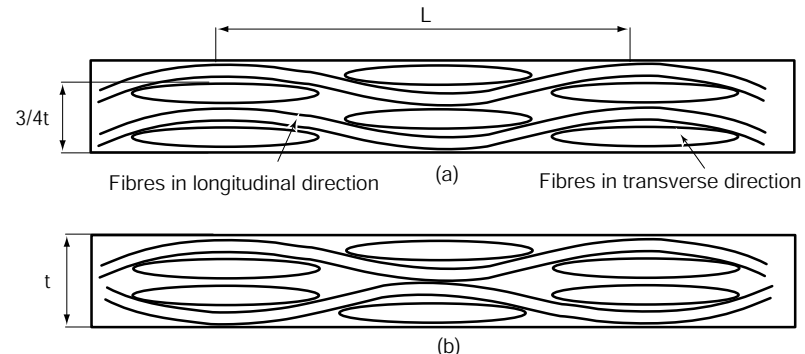

Fig. 13 Schematic cross-section of two-ply T3001K/LTM45 $[0]_{2}$ specimen; (a)Fibers in phase; (b)Fibers out of phase.

To compare results to the experimental data, the thickness of each yarn and the total thickness of the model are taken to be $0.05 \mathrm{~mm}$, and $0.2 \mathrm{~mm}$, respectively. Figure 14 shows a two-ply laminate $(0,0)$ model, where warp or weft fibers running in parallel to each 
other as in Figure 13a. Top and bottom layers are connected again rigid beam connectors at the crossover points.

Preliminary results showed that the longitudinal fibers suffered localized deformations when subject to high curvatures, yielding nonlinear behavior for bending stiffness as well as bending strains. This is due to fact that the matrix hold the fibers in place so that there is no significant relative motion of the fibers through the thickness in a cross-section. The model was then modified with minimal changes; equally spaced three-rigid-link connectors were added between two consecutive crossover points of the same warp yarn, connecting top and bottom layers. The link connectors provide a pinned rigid link between two nodes to keep the distance between the two nodes constant.

Results are given in Figures 15 and 16 for moment per unit width versus curvature and strain versus curvature for both FE model (6 by 15 ) and CLT, respectively. Again linear behavior of the model is observed. Strains at top-right corner of each yarn of $180^{\circ}$ bent model are plotted in Figure 17. The model shows plate-like behavior: outer fibers in a cross-section are subject to high strains (tension at the top and compression at the bottom), and variation of longitudinal strains along warp fibers is uniform.

Bending stiffness and minimum bending radius of the models are compared to experimental data [8] as well as to those of CLT in Table 4. The experimental data give a range for different specimen size. Note that 6 by 15 model yields less stiffness and minimum bend radius when compared to 6 by 7 model. CLT always overestimates bending stiffness and minimum bend radius. When compared to the experimental data, CLT overestimates both bending stiffness and minimum bend radius by $82 \%$ and $33 \%$, respectively, but this time FE model overestimates both bending stiffness and minimum bend radius by $38 \%$ and $15 \%$, respectively.

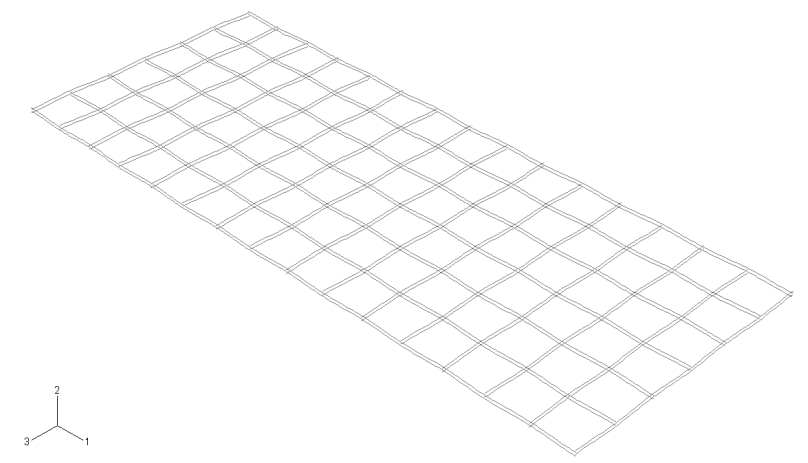

Fig. 14 Plain weave laminate model (two plies: fibers are in phase, 6 by 15 units).

The other case, in which fibers of one ply are out-ofphase with respect to the fibers of the other ply as in

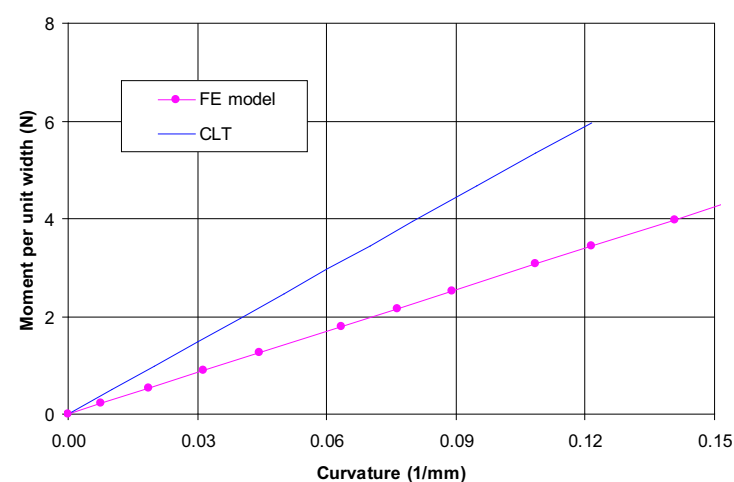

Fig. 15 Moment per unit width versus curvature (two plies: fibers are in phase, 6 by 15 units).

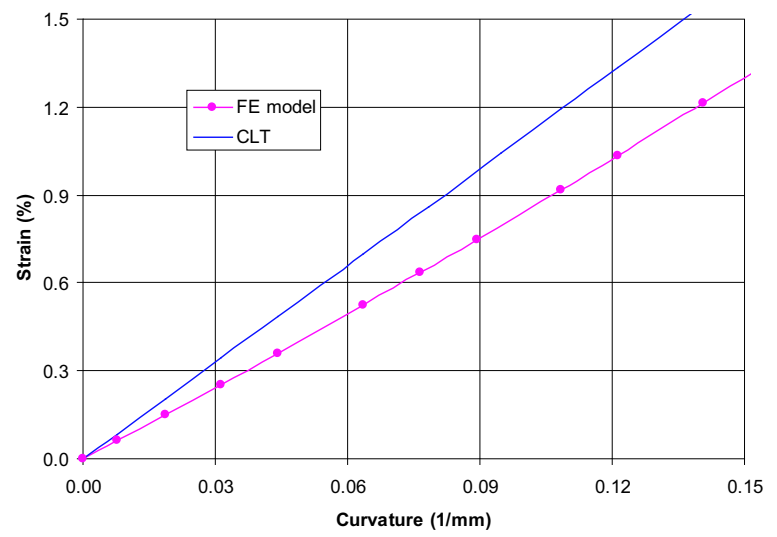

Fig. 16 Strain versus curvature (two plies: fibers are in phase, 6 by 15 units).

Table 4 Bending stiffness and minimum radius of curvature of two plies.

\begin{tabular}{|l|c|c|}
\hline \multicolumn{1}{|c|}{ Two plies (0) } & \multicolumn{1}{|c|}{$\begin{array}{c}\mathbf{D}_{\mathbf{1 1}} \\
(\mathbf{N m m})\end{array}$} & $\begin{array}{c}\mathbf{R}_{\min } \\
(\mathbf{m m})\end{array}$ \\
\hline 6 by 7 model (fibers in phase) & 31.7 & 5.89 \\
\hline 6 by 15 model (fibers in phase) & 28.3 & 5.77 \\
\hline 6 by 7 model (fibers out of phase) & 18.1 & 7.16 \\
\hline Experiment (Ref. 8) & $18.3 \sim 22.7$ & $4.76 \sim 5.26$ \\
\hline CLT & $37.4^{*}$ & $6.67^{\dagger}$ \\
\hline $\begin{array}{l}{ }^{*}: \mathrm{E}_{1} \mathrm{t}^{3} /\left(12\left(1-\mathrm{v}_{12}{ }^{2}\right)\right), \mathrm{t}=0.2 \mathrm{~mm}, \mathrm{v}_{12}=0.05 \\
{ }_{+}: \mathrm{t} /\left(2 \varepsilon_{\max }\right.\end{array}$ \\
\hline
\end{tabular}



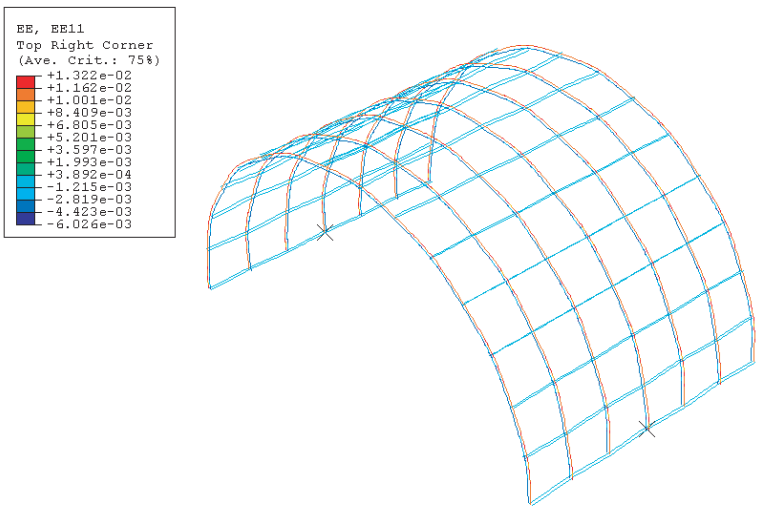

Fig. 17 Strains of $180^{\circ}$ bent model (two plies: fibers are in phase, 6 by 15 units).

Figure 13b, is also studied. Results are given in Figure 19 and 20 for moment per unit width versus curvature and strain versus curvature for both FE model ( 6 by 7 ) and CLT. When compared to the experimental data, this model yields less bending stiffness by $12 \%$ while overestimates minimum bend radius by $43 \%$. Strains at top-right corners of the beam elements of the model when $75^{\circ}$ bent are plotted in Figure 18. High strains appear at every other cross-over points in the model where the distance between upper and lower longitudinal yarns are minimum.

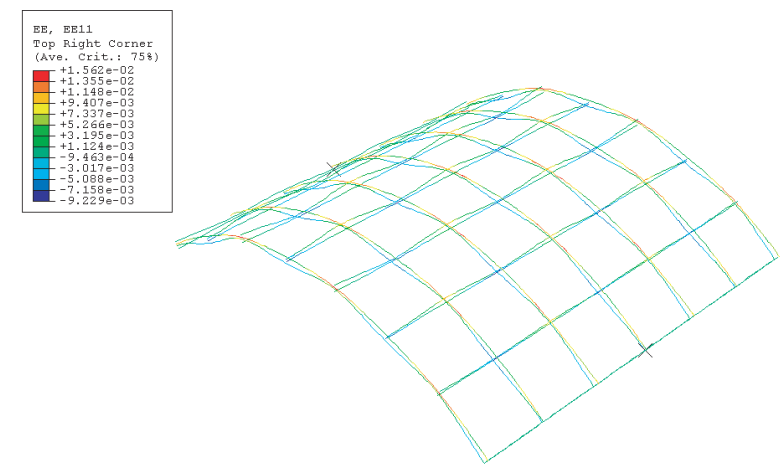

Fig. 18 Strains of $75^{\circ}$ bent model (two plies: fibers are out of phase, 6 by 7 units).

\section{Three Plies}

A three-ply model, in which fibers in a ply are in phase with the fibers of the other plies as in Figure $13 \mathrm{a}$, is considered. Results are given in Figures 21 and 22 for moment per unit width versus curvature and strain versus curvature for both FE model ( 6 by 7 ) and CLT, respectively. Linear bending behavior is observed. When compared to FE model, CLT still overestimates bending stiffness by $6.6 \%$ and yields almost same strain values ( $0.7 \%$ difference). The material behaves like almost homogenous. Strains at top-right corner of each yarn of $50^{\circ}$ bent model are plotted in Figure 23. The model shows again plate-like

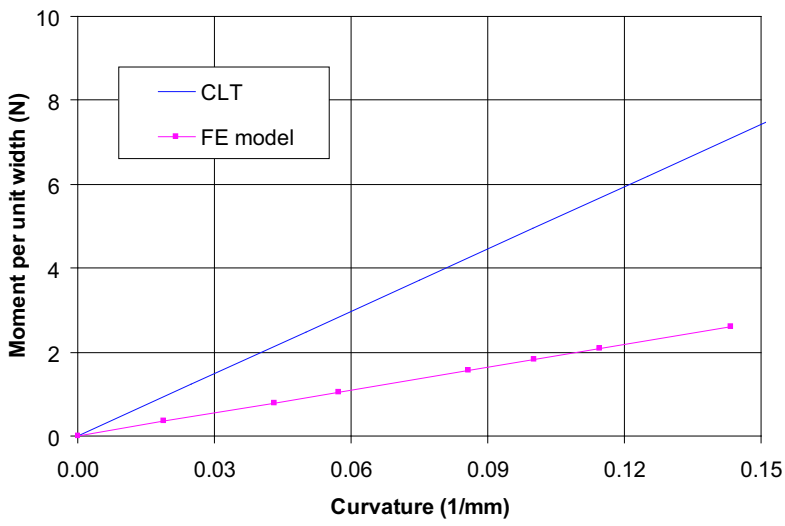

Fig. 19 Moment per unit width versus curvature (two plies: fibers are out of phase, 6 by 7 units).

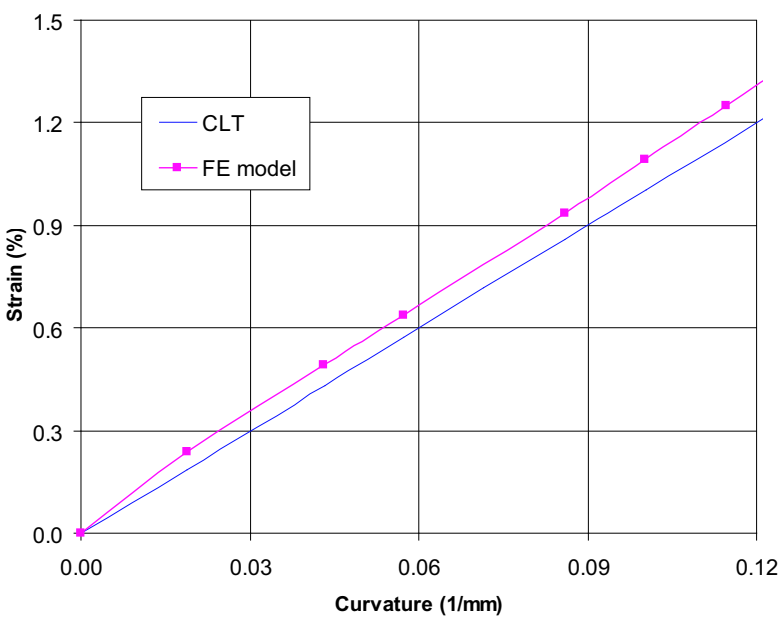

Fig. 20 Strain versus curvature (two plies: fibers are out of phase, 6 by 7 units).

behavior: outer fibers in a cross-section are subject to high strains (tension at the top and compression at the bottom), and variation of longitudinal strains along warp fibers is uniform.

Table 5 Bending stiffness and minimum radius of curvature of three plies.

\begin{tabular}{|l|c|c|}
\hline \multicolumn{1}{|c|}{ Two plies (0) } & \multicolumn{1}{|c|}{$\begin{array}{c}\mathbf{D}_{\mathbf{3}} \\
(\mathbf{N m m})\end{array}$} & $\begin{array}{c}\mathbf{R}_{\min } \\
(\mathbf{m m})\end{array}$ \\
\hline 6 by 7 model (fibers in phase) & 157.7 & 10.92 \\
\hline CLT & $168.1^{*}$ & $11.00^{\dagger}$ \\
\hline${ }^{*}: \mathrm{E}_{1} \mathrm{t}^{3} /\left(12\left(1-\mathrm{v}_{12}{ }^{2}\right)\right), \mathrm{t}=0.33 \mathrm{~mm}, \mathrm{v}_{12}=0.05$ \\
$\mathrm{t}: \mathrm{t} /\left(2 \varepsilon_{\max }\right.$
\end{tabular}

\section{Conclusions}

Micromechanical models have been developed to predict bending properties of plain weave composites. Bending behavior of one, two, and three-ply laminates is investigated. Main conclusion are as follows: 


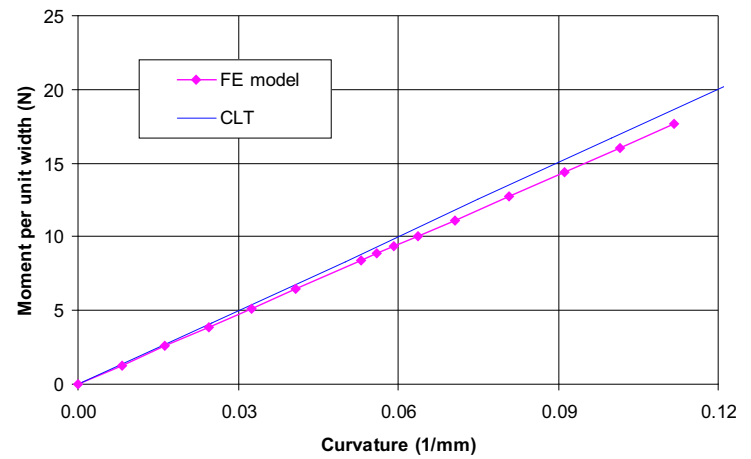

Fig. 21 Moment per unit width versus curvature (three plies).

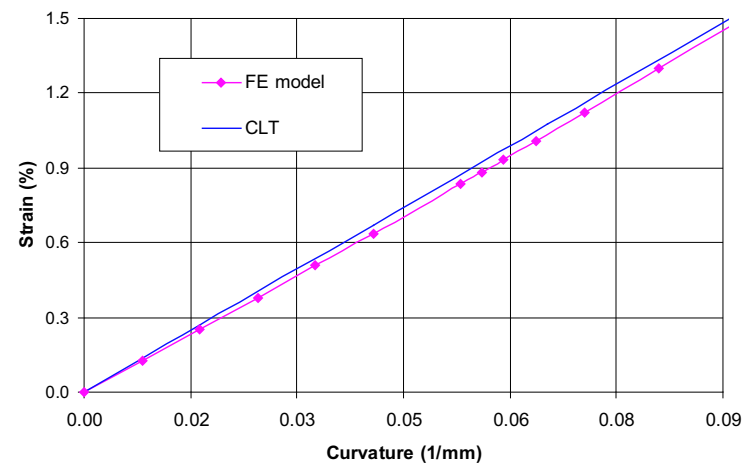

Fig. 22 Strain versus curvature (three plies).

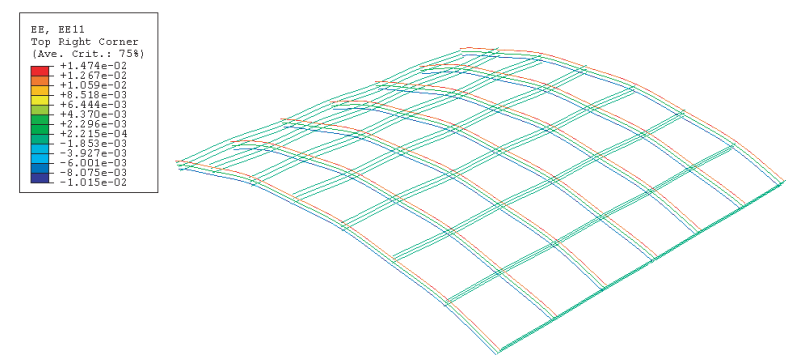

Fig. 23 Strains of $50^{\circ}$ bent model (three plies: fibers are in phase, 6 by 7 units).
- One ply: the results are in very good agrement with experimental data. When compared to experimental data, there is only $3 \%$ difference in bending stiffness and $4 \%$ difference in minimum bend radius. CLT overestimates both bending stiffness and minimum bend radius by a factor of 3.9 and 2 , respectively.

- Two-ply laminate: fiber alignment in a ply with respect to other ply plays significant role in bending behavior. Two extreme cases are studied: (i) fibers are in phase and (ii) fibers are out-ofphase. When compared the experimental data, CLT overestimates both bending stiffness and minimum bend radius by $82 \%$ and $33 \%$, respectively. When fibers are in phase FE model yields bending stiffness and minimum bend radius with $38 \%$ and $15 \%$ difference from experimental data, respectively. When fibers are out of phase FE model yields bending stiffness and minimum bend radius with $12 \%$ and $43 \%$ difference from experimental data, respectively. Note that two cases yield upper and lower bounds for the bending stiffness, and the experimental data are based on the specimens manufactured by not considering fiber alignment in micro level.

- Three-ply laminate: when compared to FE model, CLT overestimates bending stiffness by $6.6 \%$ and yields almost same strain values ( $0.7 \%$ difference). The material behaves like almost homogenous.

\section{Acknowledgement}

Discussions with Prof. Dr. Sergio Pellegrino are gratefully acknowledged.

\section{References}

${ }^{1}$ Yee, J.C.H, and Pellegrino S., Folding of Woven Composite Structures, Composites/A, Vol. 36, No. 2, pp. 273 - 278, 2005.

${ }^{2}$ Gibson, R.F., Principals of Composite Material Mechanics, McGraw-Hill Book Co., Singapore, 1994.

${ }^{3}$ Fujita A, Hamda H., and Maekawa Z., Tensile Properties of Triaxial Woven Fabric Composites, J. Composites Mater, Vol. 27, No. 15, pp. 1428 - 1442, 1992.

${ }^{4}$ Dano M.L., Gendron G., Picard A., Mechanical Behavior of a Triaxial Woven Fabric Composite, Mechanics of Composite Materials and Structures, Vol. 7, pp. 207 - 224, 2000.

${ }^{5}$ Benarcyk B.A., Arnold S.M., Micromechanics-Based Modeling of Woven Polymer Matrix Composites, AIAA Journal, Vol. 41, No. 9, pp. 1788 - 1796, 2003.

${ }^{6}$ Page B.H.L., Guild F.J., Ogin S.L., Smith P.A, Finite Element Simulation of Woven Fabric Composites, Composites: Part A, Vol. 35, pp. $861-872,2004$.

${ }^{7}$ Hibbit, Karlsson and Sorensen, Inc., ABAQUS/Standard User's Manual, Version 6.4., Pawtucket, RI, USA, 2004.

${ }^{8}$ Yee, J.C.H, and Pellegrino S., Biaxial Bending Failure Locus for Woven-Thin-Ply Carbon Fibre REinforced Plastic Structures, 46th AIAA/ASME/ASCE/AHS/ASC Structures, Structural Dynamics, and Materials Conference, Austin, Texas, AIAA-2005-1811, 18-21 April 2005. 